\section{Closed-loop linear dispersion coded eigen-beam transmission and its capacity}

\author{
D. Yang, N. Wu, L.-L. Yang and L. Hanzo
}

A flexible closed-loop multiple-input-multiple-output (MIMO) system amalgamating linear dispersion codes and eigen-beam transmission is proposed that achieves a higher capacity than the equivalent openloop scheme, at the cost of feeding back a modest number of feedback bits from the mobile to the base station using Grassmannian beamforming-vector quantisation.

Introduction: The single-stream eigen-beam transmission scheme of [1] achieves significant array gains over open-loop schemes in multipleinput-multiple-output (MIMO) single-user systems, at the cost of feeding back from the mobile station (MS) to the base station (BS) a modest number of bits generated by a Grassmannian beamformingvector quantiser. The novel contribution of this Letter is that we extend this method to support multiple data streams and hence achieve a higher capacity. Our second contribution is that we amalgamate it with novel LDCs for increasing the attainable diversity gain.

Algorithm: The system having $M$ transmit antennas and $N$ receive antennas investigated in [1] is modelled in the form of $\boldsymbol{y}=\boldsymbol{H v} \boldsymbol{s}+\boldsymbol{n}$, where $\boldsymbol{y}$ is the received signal, $\boldsymbol{H}$ is an $(N \times M)$-element complexvalued matrix representing the IID Rayleigh channel, $v$ is an $(M \times 1)$ element complex-valued beamforming vector, $s$ is the transmitted symbol at each time instance and $\boldsymbol{n}$ denotes the Gaussian noise. The beamforming vector $\boldsymbol{v}$ is chosen as the channel's eigen-vector corresponding to the highest-power eigen-value denoted as $\boldsymbol{v}_{\max }$, in order to achieve the highest possible beamforming gain.

Naturally, the channel is estimated at the MS's receiver and the resultant value of $v$ is fed back to the BS in a practical frequency-division-duplex (FDD) system from the MS using a low-rate uplink (UL) feedback channel. Hence, constructing an efficient quantiser for $\boldsymbol{v}$ becomes important. The quantiser codebook design is treated as a so-called Grassmannian line packing problem in [1], which populates the one-dimensional subspace with as high a number of quantised values as possible, while maximising their minimum distance to avoid their corruption to the immediate neighbours. This mathematical problem is well-documented and the efficiency of this codebook design is demonstrated in [1].

To increase the throughput of their design, the authors of [1] employed spatial multiplexing (SM) in their later work [2], where independent information streams are transmitted over different transmit antennas. They proposed a quantised water-filling aided full eigenbeam transmission scheme using a Grassmannian right-hand-side (RHS) singular-matrix quantiser, stating that finding a good codebook for the RHS singular-matrix quantisation problem is challenging.

Hence, in order to dispense with the RHS singular-matrix at the transmitter, the employment of linear dispersion codes (LDCs) [3] is proposed instead of SM in our study, as illustrated in Fig. 1. By setting the eigen-beamforming vectors to $\boldsymbol{v}_{1}=\boldsymbol{v}_{\max }, \boldsymbol{v}_{i}=0$ if $i \in\{2, \ldots, M\}$ and setting the power allocation values to $d_{1}=1, d_{i}=0$ if $i \in\{2, \ldots$, $M\}$, the closed-loop full eigen-beamforming scheme is simplified to a single eigen-beam scheme by assigning all power to the most dominant eigen-beam. Before carrying out these operations, the inputshaping matrix $\boldsymbol{V}_{C}$, where $\boldsymbol{V}_{C}$ represents the eigenvectors of the covariance matrix of the LDC codeword $\boldsymbol{C}$ is employed in order to spatially decorrelate the input signal so as to disperse the input energy in the most effective way across the LDC's time slots and antennas [4]. LDCs can be designed for arbitrary modulation schemes as well as for an arbitrary number of transmit or receiver antennas, and for diverse numbers of LDC time slots $T$ and data streams $Q$. Hence they are capable of satisfying diverse design criteria, such as having a higher data-rate than the SM scheme of [5] by setting $Q \geq M$. LDCs are also capable of attaining the maximum achievable diversity order or maximum open-loop capacity etc. [6]. An LDC is specified in the form of LDC(MNTQ)-(modulationscheme) (e.g. LDC(3224)-QPSK). The LDC-encoded codeword $\mathbf{C}$ is generated as: $\mathbf{C}=\sum_{q=1}^{Q} \mathbf{A}_{q} s_{q}$, where the modulated symbols of the independent data streams $s_{q}, q \in\{1,2, \ldots, Q\}$ are spatio-temporally dispersed by the LDC dispersion matrices $\boldsymbol{A}_{i}, i \in\{1,2, \ldots, Q\}$ to all transmit antennas within a codeword duration of $T$ LDC slots. As a result, all the entries in the LDC codeword are constituted by a linear combination of all modulated symbols in the vector $s$. We refer to this property as having non-separable transmit symbols (NSTS). This NSTS property allows us to simplify the water-filling aided full eigen-beam transmission to single eigen-beam transmission at a modest performance degradation, when using the Grassmannian beamforming-vector quantiser.

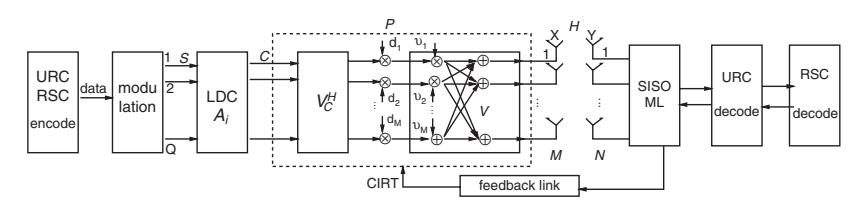

Fig. 1 Single-user MIMO system models

Results: Fig. 2 shows the discrete-input-continuous-output memoryless channel's (DCMC) MIMO capacity for an $(M=3, N=2)$-antenna system using the LDC(3224)-QPSK scheme. When using perfect channel state information at the transmitter (CSIT), the closed-loop single eigen-beam scheme achieves about $4 \mathrm{~dB}$ gain over the open-loop scheme, and it performs similarly to the closed-loop full eigen-beam scheme. When using a 3-bit instaneous beamforming vector feedback generated by a Grassmannian beamforming-vector quantiser through an error-free and delay-free feeback channel as our CSIT, the closed-loop single beam scheme achieves an approximately $2 \mathrm{~dB}$ gain over the open-loop scheme.

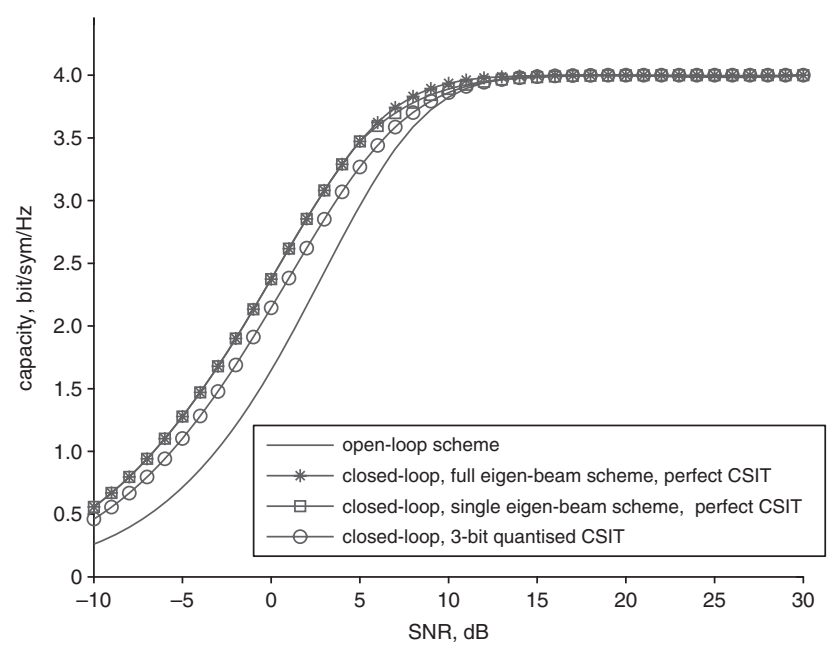

Fig. 2 DCMC capacity of $M=3, N=2$ system using LDC(3224)-QPSK and different transmission schemes

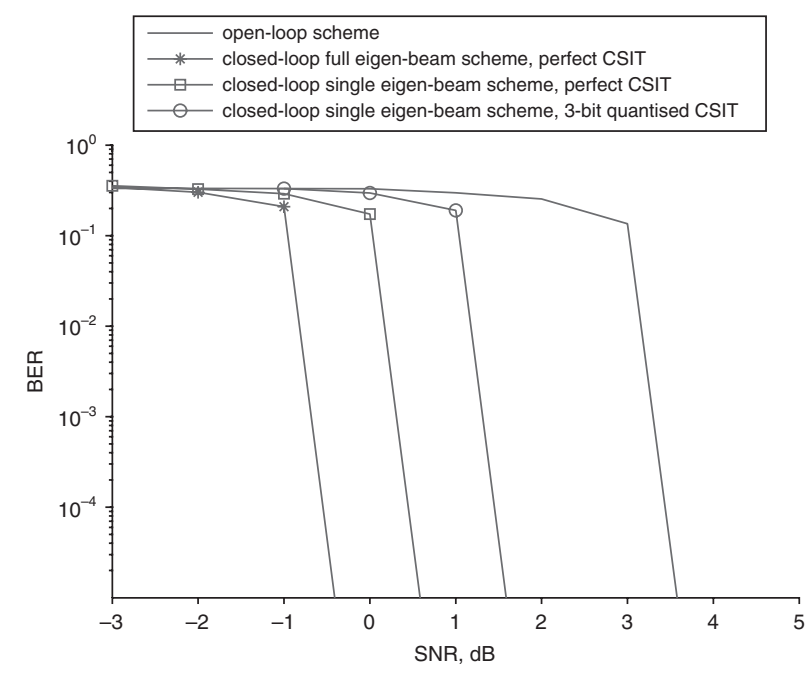

Fig. 3 BER performance of $M=3, N=2$ system using LDC(3224)-QPSK and different transmission schemes

Fig. 3 shows the achievable bit-error-ratio (BER) performance of the same system combining unitary-rate-coding (URC) [7], a half-rate recursive systematic code (RSC) RSC $(2,1,5)$ and a soft-input-soft-output 
(SISO) maximum-likelihood (ML) detector. The number of detection iterations was set to $I_{U R C}=1$ between the detector and the URC decoder and $I_{R S C}=5$ between the detector and the RSC decoder. When assuming perfect CSIT, the closed-loop full eigen-beam scheme and the closed-loop single eigen-beam scheme achieve error-free transmission at about 0 and $1 \mathrm{~dB}$, which are 4 and $3 \mathrm{~dB}$ lower than the SNR required by the corresponding open-loop scheme, respectively. Using a 3-bit quantised closed-loop single-beam scheme, a $2 \mathrm{~dB}$ gain is attainable compared to the open-loop benchmark scheme.

Conclusion: The amalgamated of LDC and single eigen-beam transmission scheme using the Grassmannian bemforming-vector quantiser is capable of providing a valuable capacity gain, while requiring a modest number of CSIT feedback bits.

Acknowledgment: The work reported in this Letter has formed part of the Core 4 Research Programme of the Virtual Center of Excellence in Mobile and Personal Communications, Mobile VCE, www. mobilevce.com, whose funding support, including that of EPSRC, is gratefully acknowledged. Fully detailed technical reports on this research are available to Industrial Members of Mobile VCE.

(C) The Institution of Engineering and Technology 2008

26 June 2008

Electronics Letters online no: 20081842

doi: 10.1049/el:20081842

D. Yang, N. Wu, L.-L. Yang and L. Hanzo (School of ECS, University of Southampton, SO17 1BJ, United Kingdom)

E-mail: dy05r@ecs.soton.ac.uk

\section{References}

1 Love, D.J., Heath, R.W., and Strohmer, T.: 'Grassmannian beamforming for multiple-input multiple-output wireless systems', IEEE Tran. Inf. Theory, 2003, 49, (10), pp. 2735-2747

2 Love, D.J., and Heath, R.W.: 'Limited feedback unitary precoding for spatial multiplexing systems', IEEE Trans. Inf. Theory, 2005, 51, (8), pp. 2967-2976

3 Hassibi, B., and Hochwald, B.M.: 'High-rate codes that are linear in space and time', IEEE Trans. Inf. Theory, 2002, 48, pp. 1804-1824

$4 \mathrm{Vu}, \mathrm{M}$., and Paulraj, A.: 'MIMO wireless linear precoding', IEEE Signal Process. Mag., 2007, 24, (5), pp. 86-105

5 Wolniansky, P.W., Foschini, G.J., Golden, G.D., and Valenzuela, R.A.: 'V-BLAST: an architecture for realizing very high data rates over the rich-scattering wireless channel'. Int. Symp. on Signals, Systems, and Electronics, Pisa, Italy, September 1998, pp. 295-300

$6 \mathrm{Wu}, \mathrm{N}$., and Hanzo, L.: 'Near-capacity irregular precoded linear dispersion codes'. IEEE Int. Conf. on Communications, Beijing, China, May 2008

7 Divsalar, D., Dolinar, S., and Pollara, F.N.: 'Serial concatenated trellis coded modulation with rate-1 inner code'. IEEE Global Telecommunications Conf., November 2000, Vol. 2, pp. 777-782 